\title{
COMPARISON OF DIFFERENT DISTANCE MEASURES FOR CLUSTER ANALYSIS OF TREE-RING SERIES
}

\author{
IGNACIO GARCÍA-GONZÁLEZ* \\ Dept. de Botánica - Univ. Santiago de Compostela, Escola Politécnica Superior - Campus de Lugo, 27002 Lugo, Spain
}

\begin{abstract}
Sixty individual ring-width series of oak (Quercus robur L.) from six sites in the northwestern Iberian Peninsula, ranging from 50 to 120 years, were grouped using hierarchical cluster analysis with different types of distance measures. Euclidean distances as well as other linkage distances based on statistics used to crossdate tree-ring series (Gleichläufigkeit and coefficient of correlation with its corresponding $t$-value) were compared. In addition, a new distance measure based on a corrected inversion of the Student's $t$ is proposed in the present paper, which takes into consideration the number of years used for series comparison. The Euclidean distances, commonly used in ecological analyses, inefficiently identified homogeneous units of trees based on their ring-width patterns. Among crossdating statistics, the correlation coefficient was more effective than Gleichläufigkeit, but the most satisfactory results were obtained when $1 / t$ was used as distance measure. Finally, these methods of cluster analysis have been implemented into a computer program for future use of the dendrochronological community.
\end{abstract}

Keywords: dendrochronology, tree ring, crossdating, oak, Quercus, Iberian Peninsula.

\section{INTRODUCTION}

Most dendroecological studies involve the comparison of tree-ring time series at different spatial scales (i.e. individual tree series or local/ regional chronologies). Commonly, when studying chronologies along an ecological gradient or when dealing with a high number of series, several techniques of multivariate analysis (e.g. principal component, cluster analysis or multidimensional scaling) can be used to simplify the interpretation of results. Among these, hierarchical cluster analysis is a classification method widely used in ecology (Gauch 1989; Legendre and Legendre 1998), which, in contrast to some other multivariate techniques, has the advantage that variables are compared pair-wise, and therefore a higher number of variables than cases does not hamper the analysis.

The use of cluster analysis in dendroecological research is not widespread, however. Occasionally, it has served as a tool to interpret the results of response functions, identifying varia-

\footnotetext{
*E-mail: bvluigg@lugo.usc.es; Fax +34 982285985
}

tions in climate-growth relationships along gradients (Fritts 1974) or in the ecological behavior at different habitats (Tessier 1989). But hierarchical classification can also be directly applied on the tree-ring series. Thus, several authors analyzed site chronologies in relation to climatic or ecological units (Wilson and Hopfmueller 2001; Piovesan et al. 2005; Koprowski and Zielski 2006) or to identify climate episodes (Bunn et al. 2005). When applied to individual tree-ring series, it allowed the identification of homogeneous groups of trees (García-González et al. 1997, 1999) or historical series (Beuting 2004), whereas Leuschner and Riemer (1989) proposed clustering as an additional tool to help dendrochronological dating. Similarly, Oberhuber et al. (1998) clustered treering sites based only on their pointer years, while Wimmer and Grabner (2000) separated homogeneous groups of anatomical variables.

Cluster analysis is fairly simple from a statistical point of view and has no formal problems when being directly applied to tree-ring data, using chronologies as variables and growth years as cases. First, it involves computing a 
matrix with the comparisons between all pairs of variables (individuals). Such comparisons can be performed using different statistical parameters and are expressed as measures of dissimilarity or distance between individuals. In a second step, individuals or groups having the shortest distances are joined together into successively larger clusters, and the final result is normally expressed as a hierarchical tree or dendrogram that facilitates the identification of groups.

This apparently simple procedure has, however, numerous variations, e.g. dissimilarity between groups can be expressed by several measures and lead to different results (Everitt 1993). Consequently, the present paper is aimed at i) evaluating how the election of a distance measure can affect the identification of homogeneous groups of tree-ring series, and ii) suggesting the most appropriate procedures for this goal, especially when using short series. For this, 60 trees growing at six different sites were classified by cluster analysis with different distance measures, and the results were compared with the expected grouping a priori. The study used individual series because they were expected to exhibit more variations and thus be more susceptible to showing differences in the methods employed.

\section{METHODS}

\section{Tree-Ring Data}

The original data set consisted of 60 individual tree-ring series of oak (Quercus robur L.). Trees were sampled at six different sites in the northwestern Iberian Peninsula (Figure 1, Table 1), selecting a subset as representative for each site (i.e. 10 series per site highly correlated with the local mean). Sites were chosen a priori to allow the observation of different levels of similarity (Figure 1). Three sites (BOO, PER and VIG) are located at medium altitude (475-700 $\mathrm{m}$ a.s.1.) within an inland mountain range; the others (EUM, BIS and ARG) lie at low altitude (200 $300 \mathrm{~m}$ a.s.1.), close to the coastline, with two of them (BIS and ARG) within the same river watershed. Thus, the analysis was essentially based on two different units (medium altitude/inland $v s$. low altitude/coastal). Within these units though, the latter includes a pair of sites that integrates a clear subgroup (BIS and ARG), whereas the former group is more heterogeneous (PER and VIG are closer, but VIG and BOO are higher and more inland, having a more continental influence). Disturbance regimes identified by the correlation between trees (Table 1) were also diverse. Trees at PER and ARG, and to a lesser extent BIS, had several asynchronous growth reductions and releases. The length of the individual series ranged from 50 to 120 years, which lies within a common range for ecological studies for this species and location.

Ring width was measured to the nearest $0.01 \mathrm{~mm}$ along two radii in each tree. Tree-ring series were detrended using a double-step standardization (Grissino-Mayer et al. 1996), i.e. fitting a negative exponential followed by a cubic smoothing spline with a $50 \%$ cut-off frequency for a 32 year wavelength. Growth indices (Fritts 2001) were computed by division, and indices of the same tree were averaged into an individual tree chronology. Autocorrelation was removed by autoregressive modelling, ensuring that mostly year-to-year variation (high frequency) was retained in the series, and also avoiding the need of correction for an effective sample size when correlating two series (Wigley et al. 1987), which would otherwise be necessary to correctly compute $t$-values.

\section{Cluster Analysis and Distance Measures}

The 60 detrended tree-ring series were compared pair-wise using different measures of linkage distance, clusters were built from the resulting dissimilarity matrix, and the results expressed as dendrograms. The grouping method was the unweighted pair-group average (UPGMA), proposed by Sneath and Sokal (1973). According to this method, the distance between two clusters is calculated as the average distance of all pairs of series between both clusters, and it works well when groups are expected to include a similar number of individuals (Legendre and Legendre 1998).

Distance measures (Table 2) were based on parameters commonly used for comparing or crossdating tree-ring series. In addition, Euclidean 


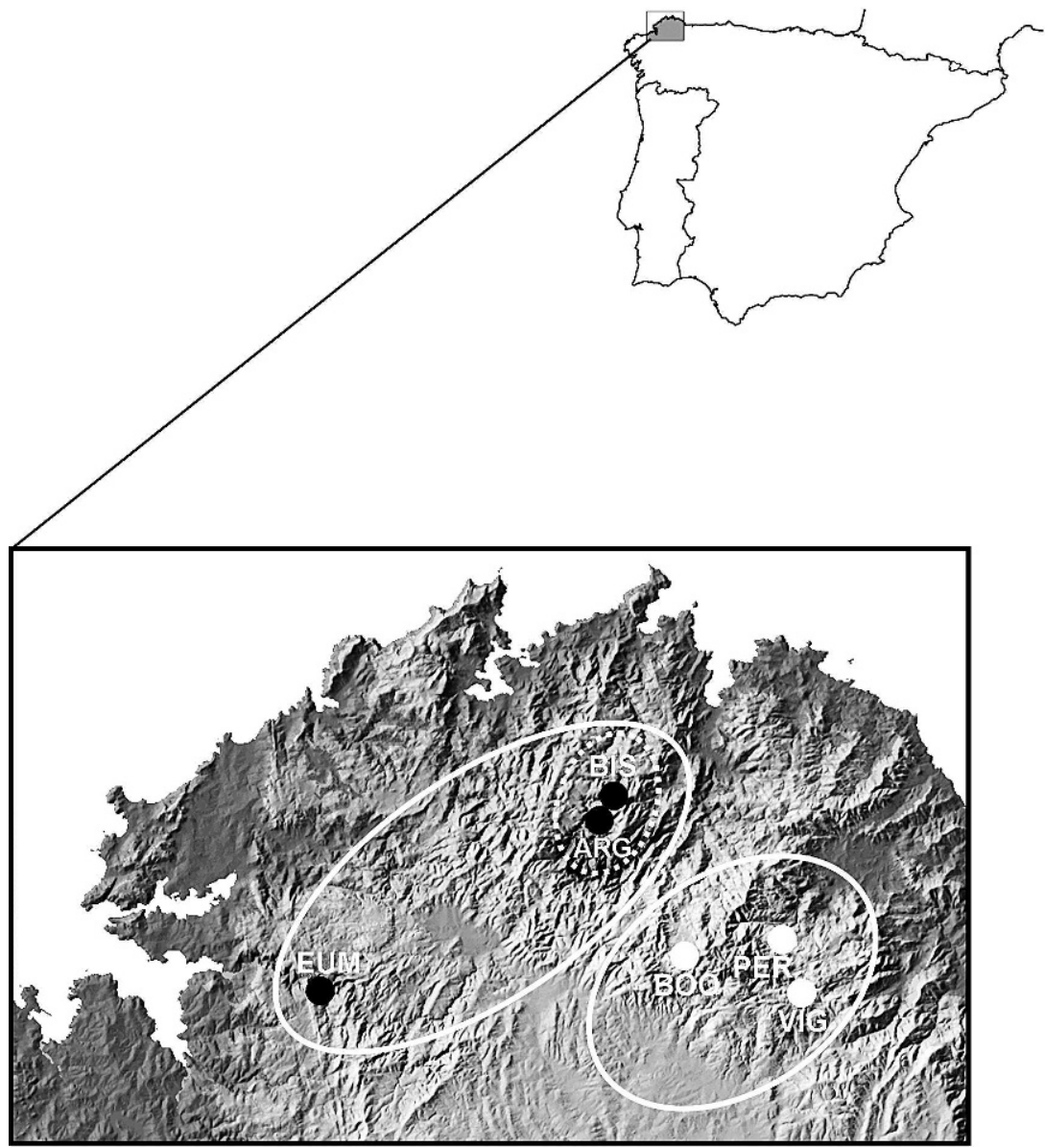

Figure 1. Location of the study sites showing the groups and subgroups established a priori.

Table 1. Sample sites selected for analysis. Mean correlation between trees (Rbt) is shown as indicator of the similarity of series within sites.

\begin{tabular}{|c|c|c|c|c|c|}
\hline Site & Code & Coordinates & Elevation & Time Interval & Rbt \\
\hline Arganzo & ARG & $\begin{array}{l}43^{\circ} 34^{\prime} 15^{\prime \prime} \mathrm{N} \\
7^{\circ} 43^{\prime} 28^{\prime \prime} \mathrm{W}\end{array}$ & $300 \mathrm{~m}$ & 1919-1997 & 0.513 \\
\hline Ribeiro do Bispo & BIS & $\begin{array}{l}43^{\circ} 34^{\prime} 48^{\prime \prime} \mathrm{N} \\
7^{\circ} 43^{\prime} 27^{\prime \prime} \mathrm{W}\end{array}$ & $300 \mathrm{~m}$ & $1886-1997$ & 0.539 \\
\hline Fragas do Eume & EUM & $\begin{array}{l}43^{\circ} 24^{\prime} 11^{\prime \prime} \mathrm{N} \\
8^{\circ} 3^{\prime} 41^{\prime \prime} \mathrm{W}\end{array}$ & $200 \mathrm{~m}$ & $1872-1998$ & 0.621 \\
\hline Fraga de Río Boó & $\mathrm{BOO}$ & $\begin{array}{l}43^{\circ} 27^{\prime} 10^{\prime \prime} \mathrm{N} \\
7^{\circ} 37^{\prime} 41^{\prime \prime} \mathrm{W}\end{array}$ & $700 \mathrm{~m}$ & $1882-1995$ & 0.642 \\
\hline O Pereiro & PER & $\begin{array}{l}43^{\circ} 28^{\prime} 42^{\prime \prime} \mathrm{N} \\
7^{\circ} 31^{\prime} 43^{\prime \prime} \mathrm{W}\end{array}$ & $600 \mathrm{~m}$ & 1899-1995 & 0.458 \\
\hline Fraga das Vigas & VIG & $\begin{array}{l}43^{\circ} 26^{\prime} 31^{\prime \prime} \mathrm{N} \\
7^{\circ} 29^{\prime} 33^{\prime \prime} \mathrm{W}\end{array}$ & $475 \mathrm{~m}$ & $1896-1995$ & 0.630 \\
\hline
\end{tabular}


Table 2. Types of distance measures used for the cluster analysis of tree-ring series.

\begin{tabular}{lcc}
\hline Statistic relating series (similarity) & Distance measure (dissimilarity) & Period of analysis \\
\hline & Euclidean distance & Common period to all series \\
Gleichläufigkeit (Glk) & $1-G l k$ & Common period to all series \\
Pearson's correlation coefficient (r) & $1-r$ & Common period to all series \\
& Corrected $1 / t$ & Common period to all series \\
& & Total overlap for every pair of series \\
\hline
\end{tabular}

distance was also considered given its frequent use for cluster analysis in ecological research. The series were compared along a common period (1945-1995, 50 years). For $1 / t$, the entire length of series was also considered, because this statistic is less sensitive to variations in sample size.

Gleichläufigkeit $(G l k)$ is a non-parametric statistic that measures the number of times two series show the same upward or downward trend in relation to the preceding year (Eckstein and Bauch 1969). However, because cluster analysis requires a dissimilarity measure, this was expressed as 1 -Glk, with Glk ranging from 1.0 (100\% agreement) to 0.0 .

The Pearson's product moment correlation coefficient $(r)$ is a parametric measure that expresses the strength of the linear relationship between two series and is the most frequent statistic used to compare tree-ring series. It can be utilized in a cluster analysis by expressing the distance as 1-r (Everitt 1993), but it is very sensitive to sample size, tending to reach higher values when series are short (Wigley et al. 1987).

Measures based on $t$-values are obtained from the above-mentioned correlation coefficient. Its significance levels can be estimated from a Student's $t$ calculated from the coefficient and sample size (Baillie and Pilcher 1973) as:

$$
t_{n-2}=|r| \sqrt{\frac{n-2}{1-r^{2}}}
$$

where $\mathrm{r}$ is correlation coefficient; $n$ is number of series.

Such $t$-values are commonly used in European dendroarchaeology (Baillie 1982) because they take into account the length of overlap (Baillie and Pilcher 1973) and thus are much more robust against variations in sample size, allowing the comparison of series of different length (Wigley et al. 1987). This is shown in Figure 2, which compares Pearson's correlation coefficient $(r)$ and its corresponding Student's $t$-value for different series length $(n)$ at a probability level of $p<$ 0.01 . Student's $t$-values are very similar after a sample size of at least $n=50$ is reached, whereas there is still an important variation of $r$.

Therefore, it seems reasonable to transform correlation coefficients into their corresponding $t$ values before clustering, but some problems arise when trying to express them as a distance measure. The computation of a corrected $1 / t$ statistic, as proposed in this paper, can help to overcome these problems.

\section{The 1/t Model}

The above-mentioned $t$-values calculated over pairs of crossdated tree-ring series express their similarities but must be converted into a linkage distance for cluster analysis (Litton and Zanoidin 1988). An easy expression would be calculated by the inversion of the $t$-value $(1 / t)$, already used by Génova (1987) to analyze the geographical difference in tree-ring chronologies. The relationship between the correlation coefficient $(r)$ and its associated $t$-value (Figure 3A) nearly overlaps until $t$ drops below 1.0. This part of the curve where the $t$-value rapidly decreases corresponds to very low correlations, which cannot be considered significant in any case $(p>$ $0.15)$. However, $t$-values change dramatically with slight differences of $r$ at these low correlation levels, and, if considered, they would modify the results of cluster analysis to a great extent because a small variation in $r$ would result in an enormous variation of $1 / t$. For this reason, the distance measure proposed in this paper consists of a correction for $t$-values lower than 1.0 assigning 


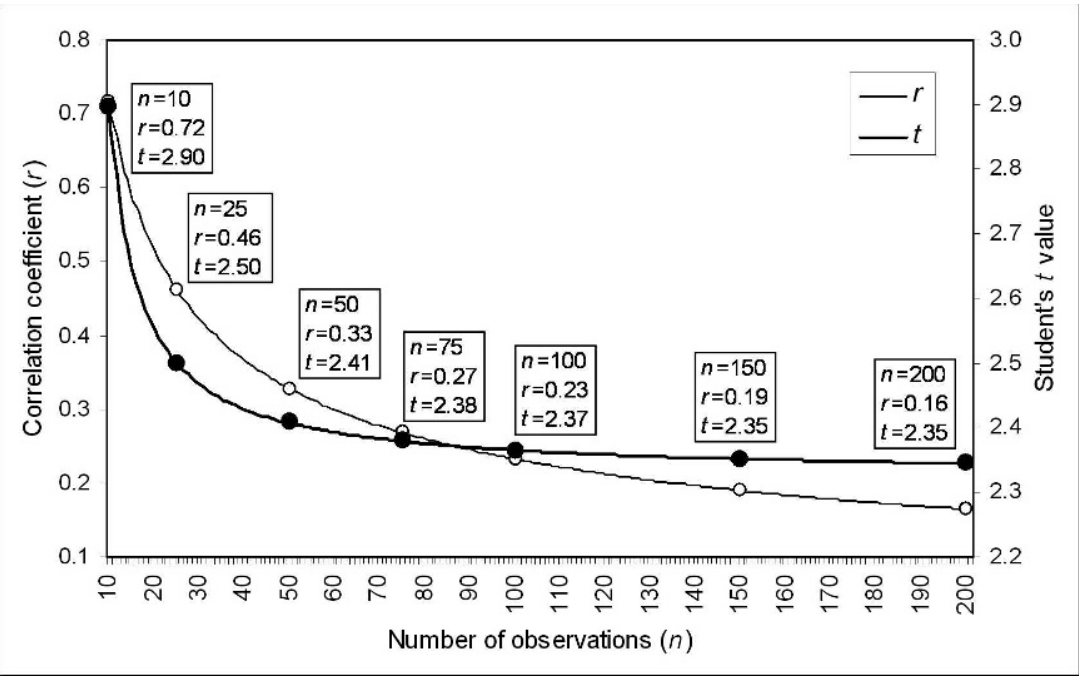

Figure 2. Comparison of the variation of the correlation coefficient $(r)$ and its corresponding Student's $t$ value when changing sample size. Values are presented for $\mathrm{p}<0.01$.

them to a value 1.0. This procedure ensures that low correlations do not influence the analysis, and that all $1 / t$ values lie between 0.0 and 1.0.

The variation of $t$-values when changing sample size is shown in Figure 2 and Figure 3B. For the latter, all curves are similar except around the inflection point, but this part corresponds to low correlations and is already corrected to 1.0, as discussed above, before clustering tree-ring series.

\section{RESULTS}

The results of using each type of distance measure were analyzed in relation to the expected grouping a priori and are shown in the corresponding dendrograms (Figures 4-8).

Euclidean distances (Figure 4) do not allow an ecological interpretation of the similarities between trees. The dendrogram shows a chainlike pattern, and sites are not sorted according to their geographic distribution, i.e. even the two main groups (coastal vs. inland sites) are not identifiable. In addition, trees from the same site (e.g. EUM or PER) are scattered at different levels of the graphs. Similarly, the result is rather disappointing if 1 -Glk is applied (Figure 5). Only trees at EUM group together and, to some extent, BOO, whereas the others follow an irregular pattern. There is no evidence of the difference among sites, and distances between individuals are much larger than those between the main clusters.

Unlike previous analyses, using Euclidean distance and 1-Glk, groups of individuals and sites are well-defined when the distance measures are based on correlations or their associated $t$-values (Figures 6-8). Figure 6 shows the result for $1-r$ and, despite the short period of analysis (1945-1994), the two main clusters are identified: trees growing in the inland sites (VIG, PER and BOO) and trees close to the coast (BIS, EUM and ARG), but still not all individuals at the same site cluster together. For the former group, VIG and PER have large distances between individuals, where $\mathrm{BOO}$ is more homogeneous, and distances among the three sites are fairly similar. For the latter, each site constitutes a single subgroup of trees, but close sites in the same river watershed (BIS and ARG) do not cluster together, even if distances between sites are not large. If the distance is otherwise expressed as $1 / t$ for the period 1945-1994 (Figure 7), the results are very similar, with the exception of one tree at ARG, classified out of its group. But the dendrogram already shows some differences with respect to $1-r$, because the distances between trees are shorter than distances between groups.

The distance based on the corrected $1 / t$ leads to the most reliable results when applied to the total time span (Figure 8) because only one tree 

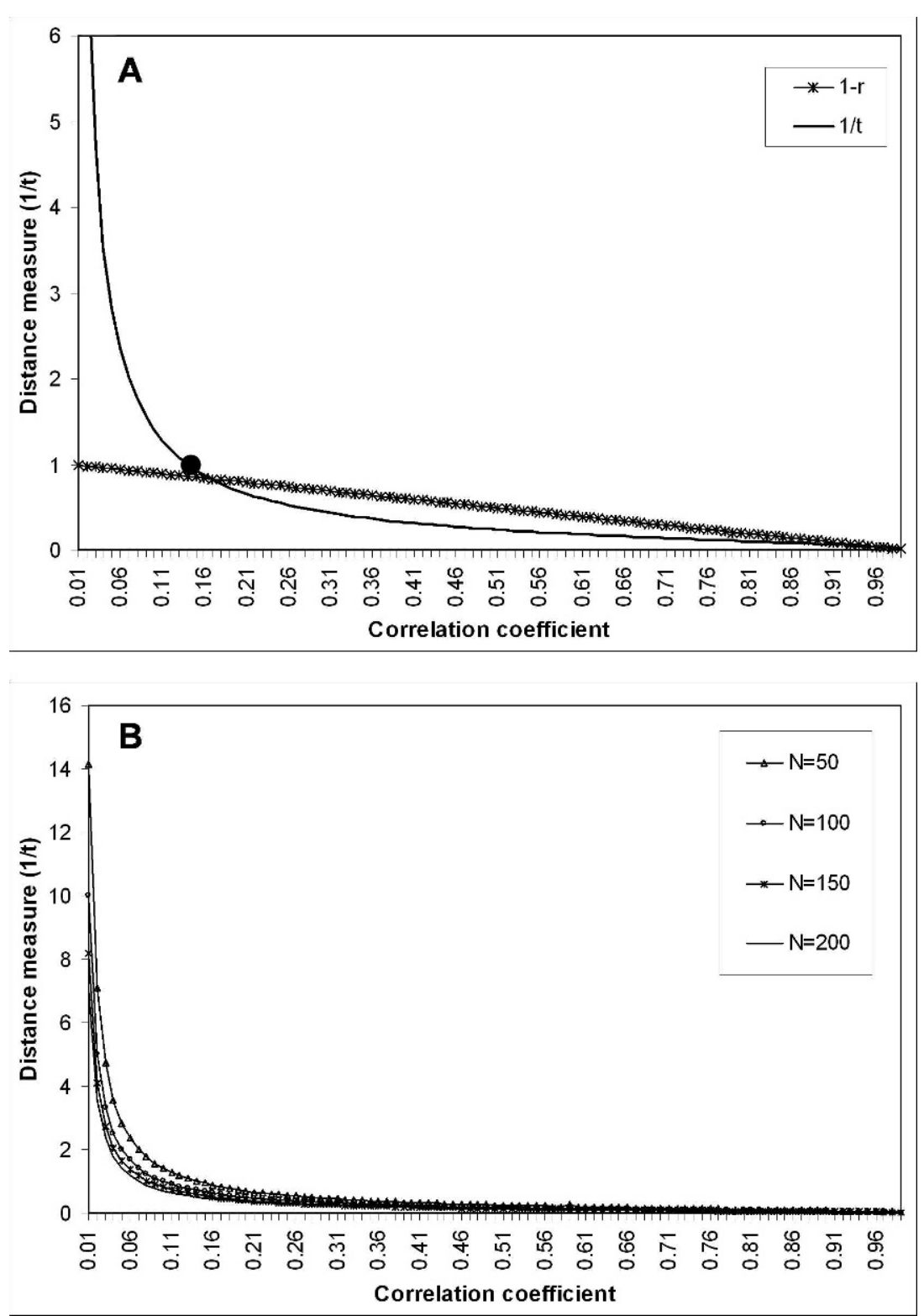

Figure 3. Variation of $1 / t$ along with the correlation coefficient $(r)$. A: Comparison between the distances $1 / r$ and $1 / t$ for a series length of 100 years (dot refers to the point where the curve of $1 / t$ is corrected to value 1.0). B: Relationship between $r$ and $1 / t$ when changing series length.

does not group to its expected cluster but to a site nearby (PER and BOO respectively). The whole dendrogram corresponds to the result expected $a$ priori: it shows two main groups (coastal and inland sites), and the clustering within each group is highly consistent with the biogeographic differences among sites. Thus, ARG and BIS are joined together, and so are BOO and VIG. In addition, distances between subgroups are short for the medium altitude sites. Heterogeneity at sites with important disturbances (such as ARG and PER) appears as larger distances between trees. Finally, distances between sites are more important than between individuals when using $1 / t$, in contrast to 


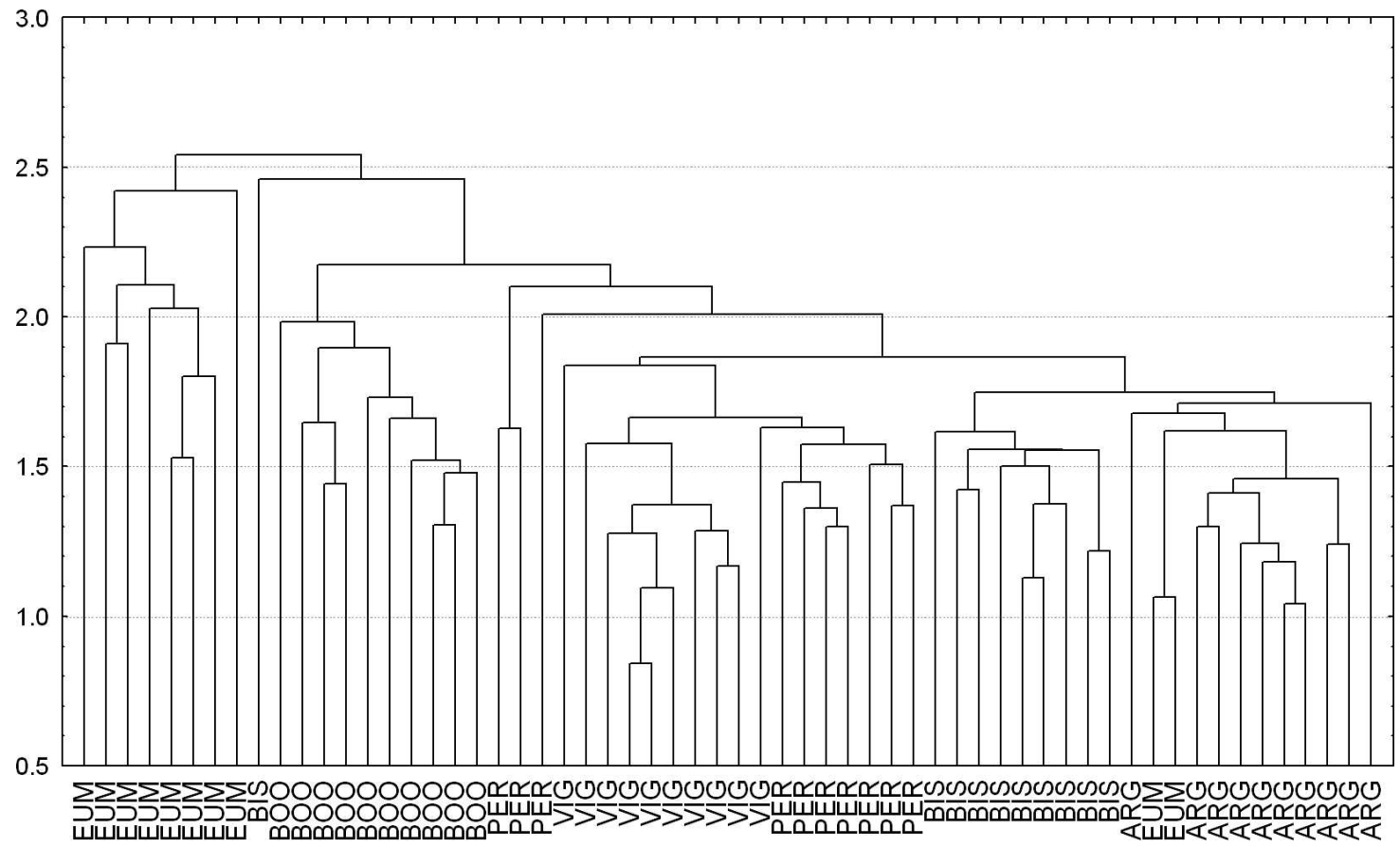

Figure 4. Dendrogram of the 60 tree-ring series using Euclidean distance. Series were analyzed along the period 1945-1994 (50 years).

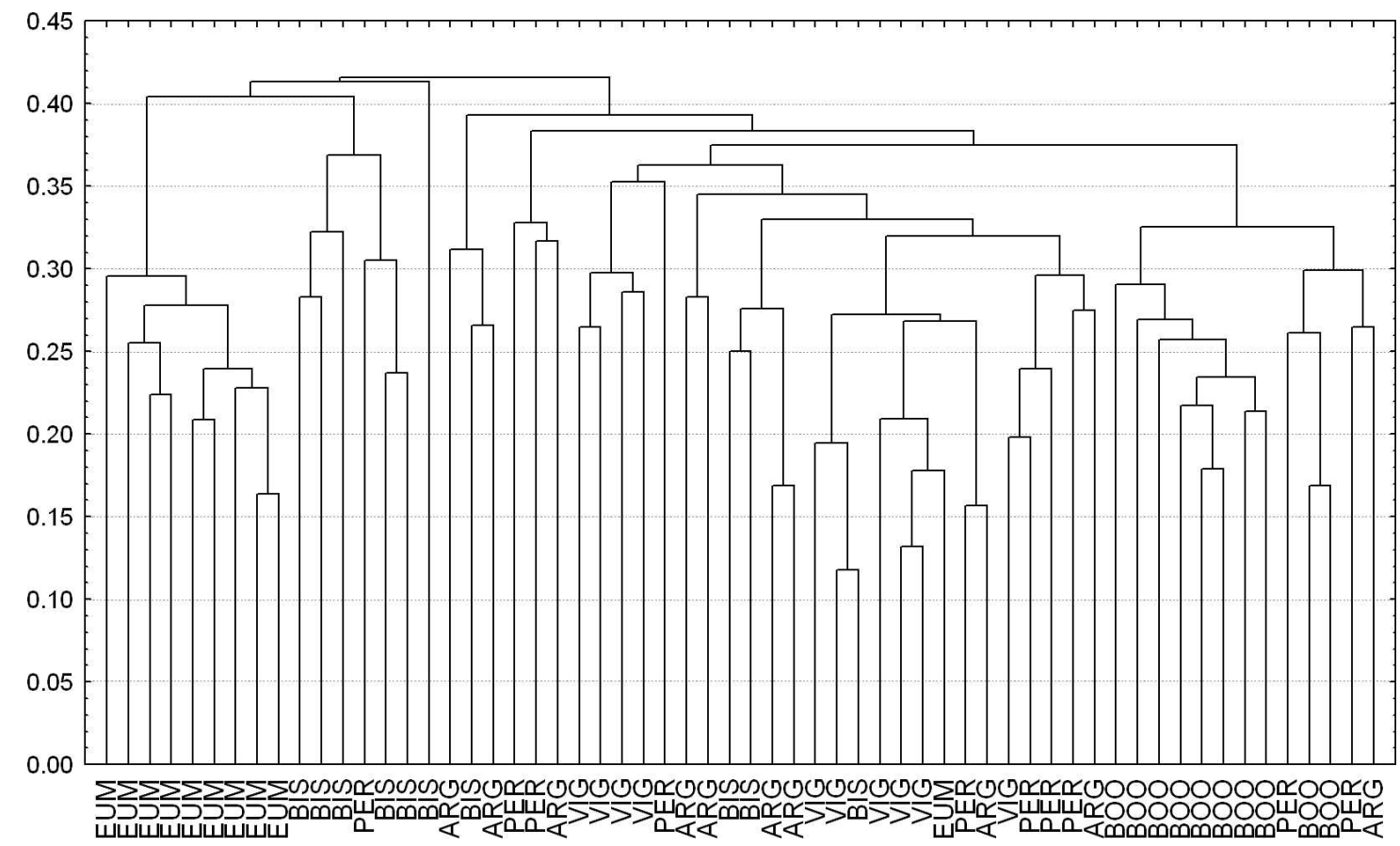

Figure 5. Dendrogram of the 60 tree-ring series sites using 1-Glk as distance measure. Series were analyzed along their entire length. 


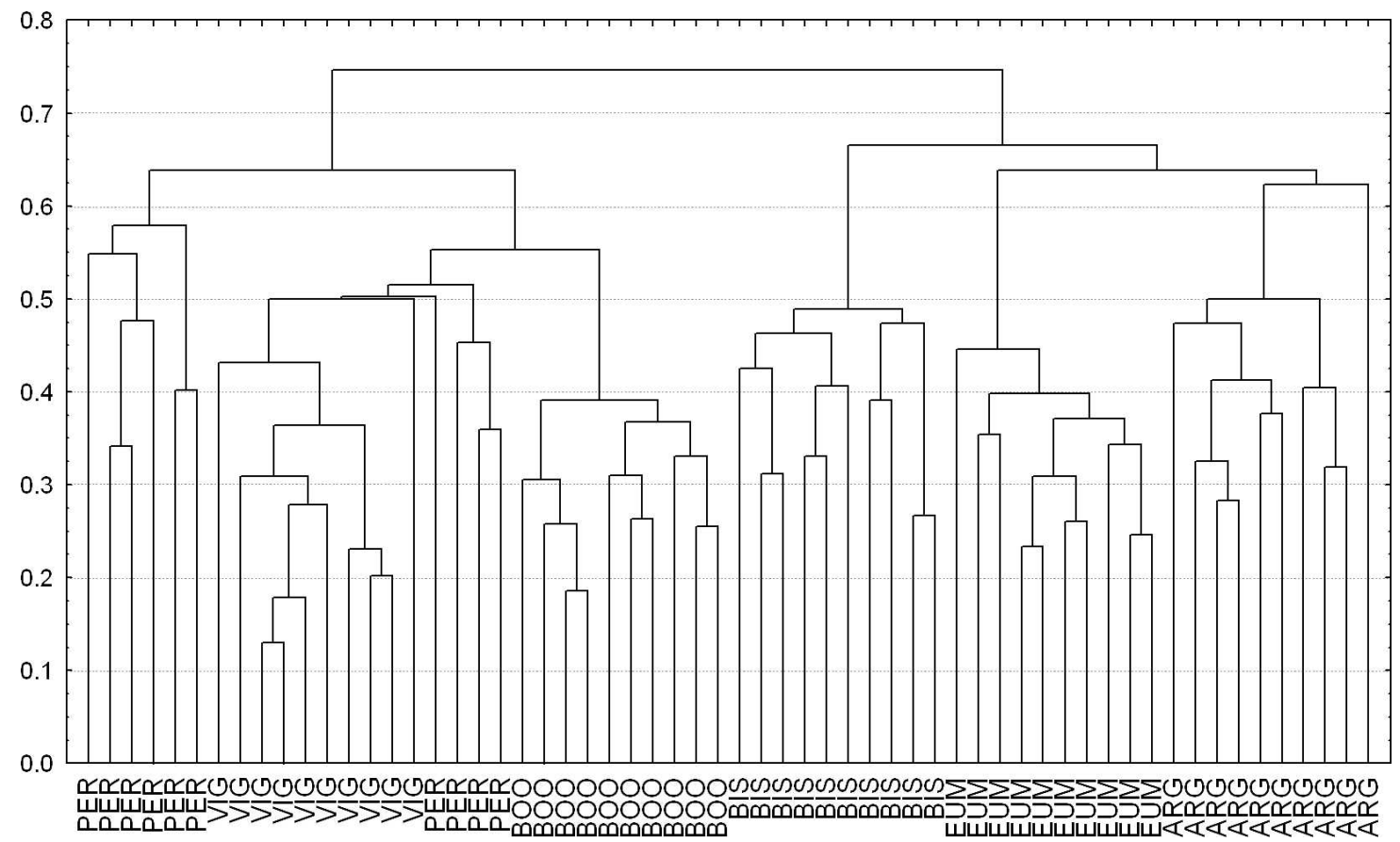

Figure 6. Dendrogram of the 60 tree-ring series using 1-r as distance measure. Series were analyzed along the period 1945-1994 (50 years).

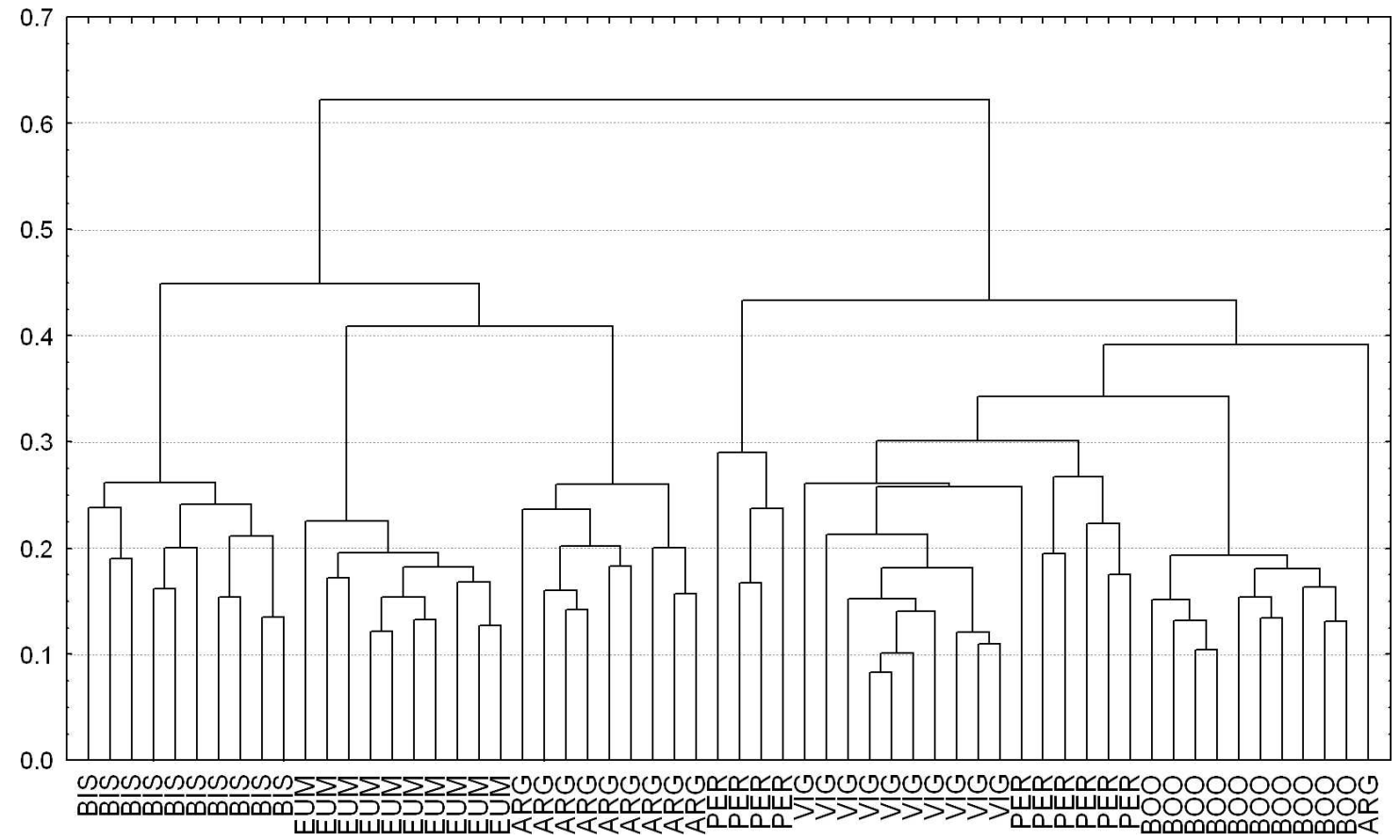

Figure 7. Dendrogram of the 60 tree-ring series using corrected $1 / t$ as distance measure. Series were analyzed along the period 1945 1994 (50 years). 


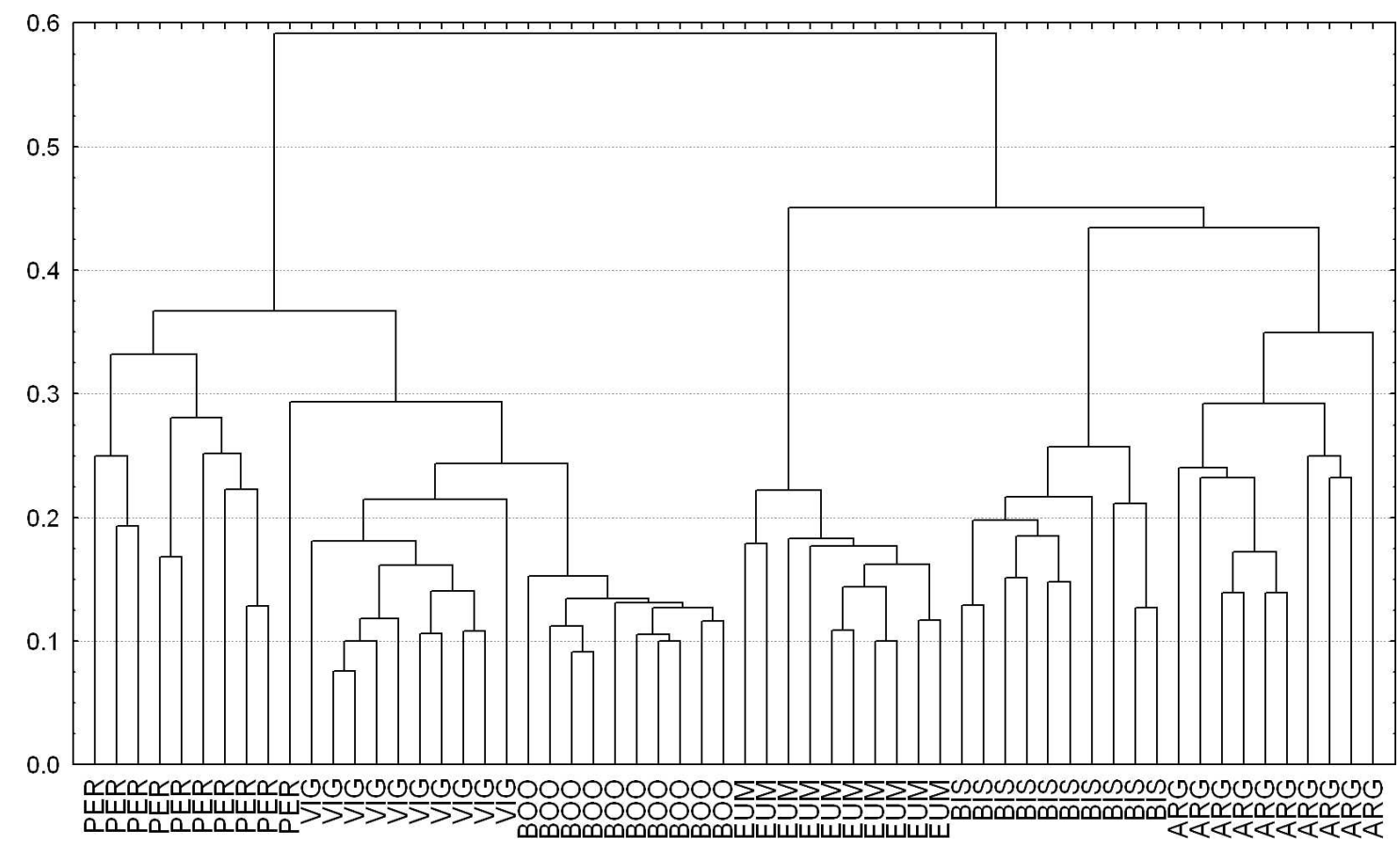

Figure 8. Dendrogram of the 60 tree-ring series using corrected $1 / t$ as distance measure. Series were analyzed along their entire length.

$1-r$, whose dendrograms were mainly determined by the distances between trees.

\section{DISCUSSION}

Cluster analysis can be a very useful tool for dendrochronological research, which can be directly applied to tree-ring series (García-González et al. 1997, 1999; Wilson and Hopfmueller 2001; Piovesan et al. 2005; Koprowski and Zielski 2006) or used for further analysis of other results, such as response functions (Fritts 1974; Oberhuber et al. 1998; Tessier 1989). With regard to the classification of tree-ring series, it is especially helpful when dealing with many chronologies or tree-ring networks. Cluster analysis can also be used to select subsamples of series, discard those that do not match, identify units or evaluate the degree of homogeneity among different subsets. The analysis starts from a matrix having the comparison between all possible combinations of tree-ring series, usually expressed as a dissimilarity or distance measure, but the above results showed that the choice of this measure can lead to very different results and should be based on statistics commonly used for crossdating. Thus, the frequently used Euclidean distances did not allow any ecological interpretation in this work. Although Oberhuber et al. (1998) succeeded in using this distance to detect populations of Scots pines with a similar response to environmental factors, they used pointer years as variables, not series of growth indices.

Groupings with Gleichläufigkeit (1-Glk) were also unsatisfactory. This parameter is often used for crossdating (Schweingruber 1988), but the results presented here might be strongly influenced by the short series length because it only considers interannual variation of sign. Therefore, 1-Glk should probably not be immediately rejected as a distance measure if longer series are compared, but could be better for site or regional chronologies than for single trees.

Linkage distances based on cross-correlations showed the most consistent results, and clusters were closer to the a priori classification. Some of these distances were successfully used in previous works for both individual trees and site chronologies. García-González et al. (1997) assessed 
species-specific responses of trees at the same site by clustering with $1-r$, and likewise Piovesan et al. (2005) and Koprowski and Zielski (2006) identified climatic patterns of tree rings, whereas Wilson and Hopfmueller (2001) classified tree-ring sites along an elevation gradient. Correlation coefficients strongly depend on series length, however, so that $1-r$ requires a fairly homogeneous time span and, if short series are present within the data set, they must be discarded or the period of analysis needs to be reduced. In this case, the series were truncated to 50 years, with some apparent negative impact in the results, i.e. the groupings were not identified so effectively. When $1-r$ was selected, most trees from the same site were correctly clustered together, the two main groups were identified (coastal vs. inland), but it failed to identify subgroups within each main group. Furthermore, clusters mainly reflected differences among individuals rather than among sites.

The main limitations imposed by the $1-r$ distances are overcome by the use of the corrected $1 / t$ statistic, which appeared to be very powerful at clustering tree-ring series. The first advantage is that series of unequal length can be compared along their entire length. In fact, when $1 / t$ was applied to the 50 -year period, the identification of groups did not differ much from that obtained with $1-r$. However, this assumes that differences through time are irrelevant, and some precautions should occasionally be taken; climatic variation or evolution of habitat could be sources of temporal instability (Tessier 1989) or the response could vary with tree age (Szeicz and MacDonald 1994; Rozas 2005) and consequently influence the results of cluster analysis. The second advantage is that $1 / t$ tends to maximize distances among groups and shorten them among individuals, which facilitates the identification of homogeneous units.

Finally, this paper showed that the corrected $1 / t$ is a very appropriate distance measure for cluster analysis of tree-ring series because it is easy to compute, does not restrict the data set to a common period and succeeds at correctly identifying patterns of similarity. A computer program for the hierarchical classification of tree-ring series, using the described methods, is available from the author on request.

\section{ACKNOWLEDGMENTS}

The author is grateful to José María Alonso and Antonio Martínez for their suggestions during the early stages of cluster analysis, and to Vicente Rozas for his comments on the manuscript. Two anonymous reviewers and the Associate Editor also contributed to improve the quality of this paper.

\section{REFERENCES CITED}

Baillie, M. G. L., 1982. Tree-Ring Dating and Archaeology. Croom Helm, London and Canberra.

Baillie, M. G. L., and J. R. Pilcher, 1973. A simple cross-dating program for tree-ring research. Tree-Ring Bulletin 33:7-14.

Beuting, M., 2004. Holzkundliche und Dendrochronologische Untersuchungen an Resonanzholz als Beitrag zur Organologie. Kessel Verlag, Remagen-Oberwinter.

Bunn, A. G., L. J. Graumlich, and D. L. Urban, 2005. Trends in twentieth-century tree growth at high elevations in the Sierra Nevada and White Mountains, USA. The Holocene 15: 481-488.

Eckstein, D., and J. Bauch, 1969. Beitrag zur Rationalisierung eines dendrochronologischen Verfahrens und zur Analyse seiner Aussagesicherheit. Forstwissenschaftliches Centralblatt 88:230-250.

Everitt, B. S., 1993. Cluster Analysis. Arnold Cop., London.

Fritts, H. C., 1974. Relationships of ring widths in arid-site conifers to variations in monthly temperature and precipitation. Ecological Monographs 44:411-440.

2001. Tree Rings and Climate. Blackburn Press, Caldwell, New Jersey.

García González, I., E. A. Díaz Vizcaíno, and A. Martínez Cortizas, 1997. Evidence for a common and a species-specific climatic response of oak and birch on a Northern Galician site (NW Spain) by means of multivariate procedures. Dendrochronologia 15:119-127.

- 1999. Dendrochronological analysis of oak (Quercus robur L., Fagaceae) in the Serra da Carba (Galicia, NW Spain): An application of cluster analysis. Nova Acta Científica Compostelana (Bioloxía) 9:171-177.

Gauch, H. G., 1989. Multivariate Analysis in Community Ecology. Cambridge University Press, Cambridge.

Génova, R., 1987. Análisis y Significado de los Anillos de Crecimiento de dos Especies Forestales: Pinus uncinata Ramond y Pinus sylvestris L. en la Península Ibérica. Dissertation, University of Barcelona (Spain).

Grissino-Mayer, H. D., R. L. Holmes, and H. C. Fritts, 1996. The International Tree-Ring Data Bank Program Library Version 2.0 User's Manual. International Tree-Ring Data Bank, Tucson, Arizona; pp. 56-58.

Koprowski, M., and A. Zielski, 2006. Dendrochronology of Norway spruce (Picea abies (L.) Karst) from two range centres in lowland Poland. Trees 20:383-390. 
Legendre, P., and L. Legendre, 1998. Numerical Ecology. Elsevier Scientific Publishing Co., New York.

Leuschner, H. H., and T. Riemer, 1989. Verfeinerte Regionalund Standortchronologien durch Clusteranalysen. Nachrichten aus Niedersachsens Urgeschichte 58:281-290.

Litton, C. D., and H. J. Zainodin, 1987. Grouping methods for dendrochronology. Science and Archaeology 29:14-24.

Oberhuber, W., M. Stumbock, and W. Kofler, 1998. Climate tree-growth relationships of Scots pine stands (Pinus sylvestris L.) exposed to soil dryness. Trees-Structure and Function 13:19-27.

Piovesan, G., F. Biondi, M. Bernabei, A. Di Filippo, and B. Schirone, 2005. Spatial and altitudinal bioclimatic zones of the Italian peninsula identified from a beech (Fagus sylvatica L.) tree-ring network. Acta Oecologica 27:197-210.

Rozas, V., 2005. Dendrochronology of pedunculate oak (Quercus robur L.) in an old-growth pollarded woodland in northern Spain: Tree-ring growth responses to climate. Annals of Forest Science 62:1-10.

Schweingruber, F. H., 1988. Tree Rings: Basics and Applications of Dendrochronology. Kluwer Academic Publishers, Dordrecht, Netherlands.
Sneath, P. H. A., and R. R. Sokal, 1973. Numerical Taxonomy. The principles and practice of numerical classification. W.H. Freeman and Co., San Francisco.

Szeicz, J. M., and G. M. MacDonald, 1994. Age-dependent tree-ring growth responses of subarctic white spruce to climate. Canadian Journal of Forest Research 24:120-132.

Tessier, L., 1989. Spatio-temporal analysis of climate tree rings relationships. New Phytologist 111:517-529.

Wigley, T. M. L., P. D. Jones, and K. R. Briffa, 1987. Crossdating methods in dendrochronology. Journal of Archaeological Science 14:51-64.

Wilson, R. J. S., and M. Hopfmueller, 2001. Dendrochronological investigations of Norway spruce along an elevational transect in the Bavarian Forest, Germany. Dendrochronologia 10:67-79.

Wimmer, R., and M. Grabner, 2000. A comparison of tree-ring features in Picea abies as correlated with climate. IAWA Journal 21:403-416.

Received 15 March 2007; accepted 8 February 2008. 